\title{
Tuberous Sclerosis Complex Renal Disease
}

\author{
Bradley P. Dixon ${ }^{\mathrm{a}} \quad$ John C. Hulbert ${ }^{\mathrm{b}} \quad$ John J. Bissler ${ }^{\mathrm{a}}$ \\ a'Division of Nephrology and Hypertension, Cincinnati Children's Hospital Medical Center, Cincinnati, Ohio, and \\ bUrologic Physicians, P.A., Edina, Minn., USA
}

\section{Key Words}

Tuberous sclerosis complex · Angiomyolipoma $\cdot$ Renal cyst - Oncocytoma - Mammalian target of rapamycin complex $1 \cdot$ Rapamycin $\cdot$ Sirolimus

\begin{abstract}
Although not as common as other genetic renal diseases such as autosomal dominant polycystic kidney disease, patients with tuberous sclerosis complex frequently have significant renal involvement. Recent revelations in the cell biology of these renal disease manifestations as well as effective therapies for tuberous sclerosis complex-related renal issues have heralded hope of improved renal survival and improved quality of life for the TSC patient. This review specifically addresses some of the major renal manifestations of this disease.

Copyright $\odot 2010$ S. Karger AG, Basel
\end{abstract}

\section{Introduction}

Tuberous sclerosis complex (TSC), an autosomal dominant genetic disorder with a birth incidence of 1:6,000, can affect virtually any organ system $[1,2]$ and all racial and ethnic groups. Although TSC has been identified by Vogt's triad (facial angiofibromas, mental retardation, and intractable epilepsy), less than $40 \%$ of affected patients have all three features [3]. TSC most often presents with neurologic symptoms, and approximately $90 \%$ of affected individuals experience seizures and about half of patients experience cognitive impairment, autism, or other behavioral disorders. Renal manifestations are the second most common findings associated with TSC, with angiomyolipomas occurring in $80 \%$ and renal cystic disease in $\sim 50 \%$ of patients. Pulmonary involvement, specifically lymphangioleiomyomatosis (LAM), is the third most common cause of TSC-associated morbidity, occurring in approximately 35\% of female TSC patients. The gender predilection in LAM is not understood.

TSC is caused by mutations in either the TSC1 gene, located on chromosome 9, or the TSC2 gene, located on chromosome 16. Typically children with TSC are born with normal kidneys but develop cystic disease and angiomyolipomas as they age. Both renal cystic disease and angiomyolipomas cause chronic kidney disease (CKD), affecting approximately one million patients with TSC worldwide. Renal disease poses a significant burden on patients with TSC because of the relentless progression, morbidity, and mortality of CKD. In fact, using death certificate data Shepherd et al. [4] identified renal failure as the leading cause of death in their adult patients at the Mayo Clinic.

\section{KARGER}

() 2010 S. Karger AG, Basel

Fax +4161306 1234

E-Mail karger@karger.ch

www.karger.com
Accessible online at: www.karger.com/nee
John J. Bissler

Division of Nephrology and Hypertension

Cincinnati Children's Hospital Medical Center, MLC 7022

3333 Burnet Avenue, Cincinnati, OH 45229-3039 (USA)

Tel. +1 513636 4531, Fax +1 513636 7407, E-Mail john.bissler@cchmc.org 

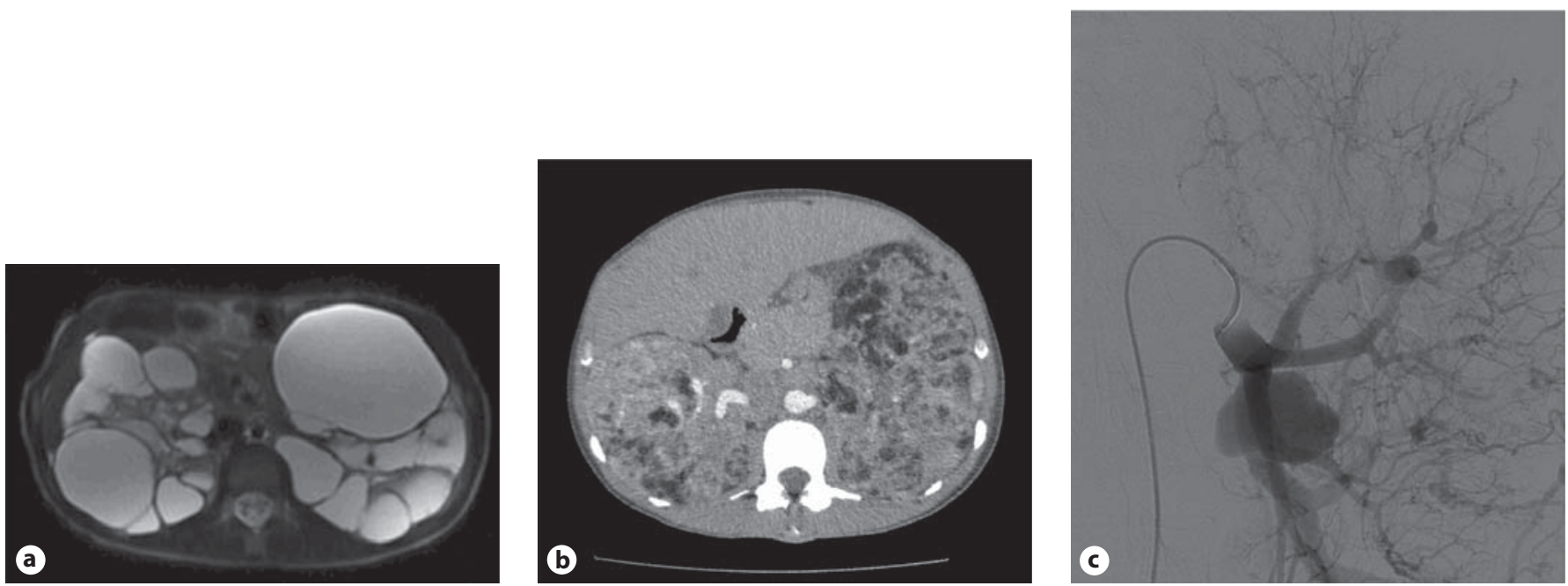

Fig. 1. Important renal manifestations of TSC. a Polycystic renal disease demonstrated on the fast spin echo $\mathrm{T}_{2}$ fat-suppressed MRI. b Significant bilateral renal angiomyolipoma burden demonstrated on the CT scan with contrast. c Angiography reveals larger and proximal aneurysmal burden.

\section{Renal Cystic Disease in TSC}

Clinically detectable renal cystic disease occurs in approximately $50 \%$ of patients with TSC associated with either the TSC1 or TSC2 gene [5-7]. TSC patients can have a severe very early-onset polycystic phenotype (fig. 1a) associated with deletions involving adjacent TSC2 and PKD1 genes on chromosome 16p13 and accounting for about $2 \%$ of TSC patients [8]. Renal cystic disease can also be microcystic, undetectable by imaging studies. Such cysts are reported to occur from all parts of the nephron, including the glomerulus [9]. Development of renal cystic disease may accelerate following acute kidney injury as it does in animal models [10]. This acceleration could have significant clinical consequences, as TSC patients have unique risk factors for acute kidney injury including use of certain anticonvulsant and nonsteroidal anti-inflammatory medications as well as from rhabdomyolysis and hypoxia induced by prolonged seizures [11].

\section{Renal Angiomyolipomas in TSC}

Angiomyolipomas, the prototype of the PEComa family of tumors, exhibit immunoreactivity for both melanocytic markers (as detected by the HMB- 45 and melaninA antibodies) and smooth-muscle markers (actin and desmin). All components of angiomyolipomas, including the vascular cells, immature smooth-muscle-like spindle cells, epithelioid cells, and fat cells contain somatic mutations that, combined with their germline mutation, render the cells deficient in either tuberin or hamartin. Presumably, this deficiency disrupts the integrated control of cell growth leading to the angiomyolipoma [12]. A crosssectional study of TSC patients revealed an increase in angiomyolipomas during childhood and adolescence that then stabilized throughout adulthood [13]. In a longitudinal study of children with TSC, 55\% of children (mean age 6.9 years) had some type of renal abnormality, and at follow-up $80 \%$ (mean age 10.5 years) had abnormalities [6], with the most common form of involvement being angiomyolipomas. Based on their findings, the authors concluded that renal involvement begins in infancy and increases with age.

Angiomyolipomas significantly affect the lives of TSC patients because these lesions are at risk for hemorrhage and can invade adjacent normal renal parenchyma (fig. 1b) leading to chronic kidney disease and even endstage renal disease. In addition to macroscopic disease, kidney tissue that is radiologically normal may, on cut section, contain both microscopic angiomyolipomas and cysts. These findings beg the question that such microscopic lesions may grow and become identifiable as the patient ages. The vascular component of larger angiomyolipomas frequently develop aneurysms (fig. 1c) that can rupture causing the hemorrhage [14-17]. The hemorrhage risk of renal angiomyolipomas in TSC patients is between 25 and $50 \%[18,19]$, and between $20-30 \%$ of pa- 
tients with hemorrhages present to the emergency room in shock [20]. The hemorrhage risk is significantly increased for aneurysms larger than $5 \mathrm{~mm}$ [21].

According to traditional urological tenets, if doubt exists regarding the nature of a renal lesion in routine practice, then nephrectomy is justified. Because familiarity with the renal manifestations of TSC is generally only found in specialized TSC centers, TSC patients with suspected retroperitoneal hemorrhage or atypical renal lesions may undergo elective or emergent, but most often avoidable, nephrectomy in centers with limited TSC experience. Such procedures hasten the requirement for renal replacement therapy. Considering the relentless progression of CKD and the inherent increased risk in morbidity and mortality, these lesions pose a significant burden for patients with TSC [22]. Given the frequent existence of microscopic angiomyolipomas, the most logical intervention is to treat hemorrhage, or reduce its risk, and not to actually remove the angiomyolipoma. It is critical to note that due to the bilateral nature of the renal lesions in TSC, and in order to preserve functional renal mass, nephrectomy should not be undertaken without a very careful risk-benefit analysis. The current standard of care to both control active bleeding as well as to prevent lesions with aneurysms from bleeding in the future is embolization [15, 23], and corticosteroid therapy can greatly reduce the subsequent postembolization syndrome [15]. Although embolization is preferable, surgical intervention may be contemplated in selected patients who have cortical or exophytic lesions that have not hemorrhaged. The goal of surgical therapy, as it is for embolization, is to do the least invasive procedure directed at a specifically targeted lesion, with the minimum risk to the remainder of the kidney. Less invasive surgical methods such as laparoscopy have reduced the overall impact of the surgery, and partial nephrectomy or ablative therapy with cryotherapy or radiofrequency treatment can be acceptable treatment options in such selected patients by properly trained urologists.

\section{Fat-Poor Angiomyolipoma}

Fat-poor lesions in the TSC patient raise significant concern, in part because of their potentially aggressive nature in non-TSC patients and in part due to the lack of reliable information about such lesions in the TSC population. Because ultrasound is exquisitively sensitive at detecting adipose tissue, it has been used as a simple and reliable imaging modality to detect angiomyolipomas.
Such sensitivity to adipose tissue may have induced an ascertainment bias such that many clinicians believe that all angiomyolipomas must have sonographically detectable adipose tissue. Most often, solid lesions in the kidney of a patient with TSC are fat-poor angiomyolipomas, but can also rarely be oncocytomas or renal cell carcinomas. In addition to utilization of better imaging techniques to detect adipose tissue, the use of angiomyolipoma growth rate has been introduced as a possible method to distinguish more ominous lesions such as renal cell carcinomas from fat-poor lesions [24], although there may be growth rate disparities as fat-poor lesions are not as easily detected by ultrasound. Careful observation over a selected time frame should determine whether the lesion is actually enlarging, and if concern still exists based on growth rate (a small minority) and tissue diagnosis is essential, then less invasive surgery with biopsy and renal preserving therapy can be considered.

Fat-poor TSC-associated angiomyolipomas can consist of predominantly spindle cells, epithelioid cells [25, 26] or vascular elements [27-29]. The contribution of each of the different cell components can vary from lesion to lesion, and some lesions can exhibit a dominant cell type $[30,31]$. Although very rare for the TSC patient population, epithelioid angiomyolipomas can exhibit an aggressive phenotype [32], recur after resection and even be fatal $[25,33,34]$. While such case reports have been published, a case series of 15 patients recently demonstrated the course may be quite benign [35], supporting that a more deliberate approach using repeat imaging may be used.

The typical hematoxylin and eosin appearance of the epithelioid variant of angiomyolipoma is very similar to that of the RCC [36], and the preponderance of RCC reported in TSC patients has not been studied in sufficient detail to exclude misclassification of an atypical angiomyolipoma such as an epithelioid variety [37]. The incidence of renal cell carcinoma (RCC) in TSC patients is estimated to be significantly less than $2 \%$. Immunohistochemical studies aimed at detecting HMB-45 and melanin A, diagnostic markers for angiomyolipoma, are extremely useful for differentiating between RCC and the epithelioid variety of angiomyolipoma in TSC.

\section{Hypertension}

Hypertension control in the TSC patient is critical given that the majority of patients have CKD. Renal cystic disease is a significant risk factor for hypertension in this 
population and responds very well to angiotensin-converting enzyme inhibitors and angiotensin receptor blockers. Despite a significant burden of renal parenchymal abnormalities observed by imaging, renal function can be amazingly preserved. This functional preservation in the face of significant imaging abnormalities is in part due to coalescence of angiomyolipomas rather than growth of a single lesion. Furthermore, though not as frequently encountered, adrenal corticotropic hormone therapy to control infantile spasms can also induce hypertension, and this mechanism of elevated blood pressure can be handled with diuretics with or without other antihypertensive agents.

\section{Nephrolithiasis}

Patients with TSC can be predisposed to nephrolithiasis both because of treatments for their disease manifestations as well as the renal disease manifestations themselves. Drugs like topiramate have proven to be a very effective therapy for some forms of TSC-associated epilepsy by enhancing GABA-activated chloride channels and inhibiting excitatory neurotransmission. Relevant to the kidney, topiramate inhibits carbonic anhydrase, particularly subtypes II and IV, leading to decreased citrate excretion and subsequently increased risk of nephrolithiasis. Alternatively, the seizure frequency of some TSC patients responds well to the ketogenic diet. The associated diet-stimulated hypercalciuria and hypocitraturia synergize to increase the risk of nephrolithiasis, and this effect is further facilitated by the resulting decrease in uric acid solubility caused by the low urine $\mathrm{pH}$. Another nephrolithiasis risk factor is significant renal cystic disease, as disruption of distal tubular function by a significant cyst burden leads to hypocitraturia. Fortunately, successful therapy for nephrolithiasis in the TSC population is relatively simple regardless of the risk factors and involves adequate hydration and citrate supplementation.

If surgical intervention for nephrolithiasis is needed, there are some required TSC-specific precautions. Traditional surgical treatment of nephrolithiasis includes minimally invasive procedures such as extra-corporeal shockwave lithotrypsy (ESWL), percutaneous nephrolithotomy (PCNL), and ureteroscopic stone removal. ESWL requires large numbers of shock waves, which may cause renal subcapsular hematoma formation, and PCNL involves accessing by puncture and dilation of the renal collecting system via the renal parenchyma. In the TSC kidney, with the potential for hemorrhage well known, such approaches may pose an unacceptable risk. Ureteroscopic stone removal is therefore likely the best option. This can be achieved using delicate flexible instruments such as stone baskets to remove small stones, or a laser fiber to direct pulsed laser energy that will fragment stones without the risk of hemorrhagic effects associated with ESWL.

\section{New Insights into Abdominal Imaging for the TSC Patient}

The relative amount of each of the adipose, spindle cell, and vascular tissue components varies in each angiomyolipoma, with a minority (4.5\%) of these tumors being classified as minimal fat or fat-poor angiomyolipomas, as they contain only microscopically detectable fat [38]. Using a combination of grey-scale and power Doppler ultrasonographic modes, Jinzaki et al. [39] evaluated a cohort of patients with small $(1.5-3 \mathrm{~cm})$ renal lesions consisting of renal cell carcinomas, angiomyolipomas, oncocytomas, and pseudotumors. Grey-scale ultrasound exhibited an extremely poor diagnostic accuracy (21\%) for angiomyolipomas, though the combination of gray-scale and power Doppler ultrasound increased the diagnostic accuracy. Despite the fact that fat-poor angiomyolipomas contain no macroscopic focus of fat by ultrasound or CT scans, these tumors histologically contain an average of $4.1 \%$ fat [40].

Magnetic resonance imaging is a very effective means of detecting both the macroscopic and microscopic adipose components of angiomyolipomas, and is especially successful in the identification of minimal fat angiomyolipomas. The basic approach has been to assess the difference between fat-suppressed and non-fat-suppressed $\mathrm{T}_{2}$ weighted sequences to locate fat within a mass. This approach is necessary because high $\mathrm{T}_{2}$ signal within a renal mass is not diagnostic for an angiomyolipoma and can be seen with both renal cell carcinoma and hemorrhagic cysts. Comparing the fat-suppressed and non-fat-suppressed sequences can identify the adipose component and reduce the concern about renal cell carcinoma in a patient with tuberous sclerosis.

In addition, utilization of MR imaging artifacts such as in-phase/out-of-phase techniques can assist in identifying fat in fat-poor lesions [41]. Israel et al. [42] assessed the use of the opposed-phase chemical shift technique to diagnose renal angiomyolipomas, and reported that all of their twenty-three angiomyolipomas could be correctly identified, though one RCC was mischaracterized as an 
angiomyolipoma. Given the differences in the sensitivity of the various imaging modalities, it is also critically important to recognize that switching from ultrasound to either CT or MRI may reveal significantly more involvement especially by a fat-poor component than at first appreciated. As such, this does not necessarily indicate a sudden accelerated growth of a lesion, but merely the resultant detection differences observed by a change in imaging modality.

While MRI offers a superior method to investigate and monitor renal involvement in TSC, there are some aspects of TSC that can preempt the use of abdominal MRI. Vagal nerve stimulators may be implanted in an effort to help control seizure activity. Unfortunately, the presence of a vagal nerve stimulator puts the patient at a low but defined risk for inductive heating, pain and tissue damage, and therefore these patients are not eligible for abdominal MRI in most centers. If a solid-appearing lesion is detected and MRI is not possible, CT imaging is a reasonable alternative, and if considerable concern remains, PET scans can be helpful because angiomyolipomas are generally not PET avid $[43,44]$.

\section{Possible Novel Therapies}

The TSC gene products hamartin and tuberin form a complex that integrates cellular signaling inputs such as growth factors, genomic integrity, cellular energy supply and growth substrate availability to gate growth and proliferation, but can affect cell cycle arrest, senescence, autophagy or cell death. The mammalian target of rapamycin complex 1 (mTORC1) is a downstream target of the hamartin/tuberin complex, and use of an mTORC1 inhibitor such as sirolimus is likely to serve as targeted therapy and ultimately affect the angiomyolipoma volume. The results of a clinical proof-of-principle trial suggest that sirolimus and analogues may be beneficial in controlling the growth of TSC angiomyolipoma [45]. After taking sirolimus for 12 months, the mean $( \pm \mathrm{SD})$ angiomyolipoma volume decreased to $53 \pm 27 \%$ of the baseline volume $(\mathrm{p}<0.001)$. However, at 24 months $(12$ months after discontinuation of rapamycin) the mean volume had increased back to $86 \pm 28 \%$ of the baseline volume ( $\mathrm{p}=0.005)$. These results led to multicenter placebo-controlled trials for angiomyolipomas (ClinicalTrials.gov identifier NCT00790400), and therapeutic trials using mTORC1 inhibitors are also underway for autosomal dominant polycystic kidney disease (NCT00346918 and NCT00491517).

\section{Conclusions}

Although previously TSC-associated renal lesions such as angiomyolipomas were handled predominantly by surgical intervention, the current management of TSC patients with these lesions has experienced a paradigm shift necessitating nephrology input to help delay progression of chronic kidney disease. Such immediate involvement includes helping to manage the sequelae of CKD in such patients and to facilitate and manage the specific care of the renal cystic and angiomyolipoma disease. Future pharmacologic therapies aimed at renal cyst and angiomyolipoma abatement as well as preventive therapies will likely become part of the nephrologist's care plan for these complex patients.

\section{Acknowledgments}

The authors would like to thank Christopher Kingswood, MD, and Janaka Wansupora, $\mathrm{PhD}$, for their thorough critique and helpful suggestions. Support for this work came from the Department of Defense (TS050008) and the NIH (DK061458).

\section{References}

1 Crino P, Nathanson K, Henske E: The tuberous sclerosis complex. N Engl J Med 2006; 355:1345-1356.

12 Yates J: Tuberous sclerosis. Eur J Hum Genet 2006; 14:1065-1073.

3 Curatolo P: Tuberous Sclerosis Complex: From Basic Science to Clinic Phenotypes. London, Mac Keith Press, 2003.

4 Shepherd CW, Gomez MR, Lie JT, Crowson CS: Causes of death in patients with tuberous sclerosis. Mayo Clin Proc 1991;66:792-796.
5 Dabora SL, Jozwiak S, Franz DN, et al: Mutational analysis in a cohort of 224 tuberous sclerosis patients indicates increased severity of TSC2, compared with TSC1, disease in multiple organs. Am J Hum Genet 2001;68: 64-80.

6 Ewalt DH, Sheffield E, Sparagana SP, Delgado MR, Roach ES: Renal lesion growth in children with tuberous sclerosis complex. J Urol 1998;160:141-145.
7 Rakowski SK, Winterkorn EB, Paul E, Steele DJ, Halpern EF, Thiele EA: Renal manifestations of tuberous sclerosis complex: incidence, prognosis, and predictive factors. Kidney Int 2006;70:1777-1782.

8 Sampson JR, Maheshwar MM, Aspinwall R, et al: Renal cystic disease in tuberous sclerosis: role of the polycystic kidney disease 1 gene. Am J Hum Genet 1997;61:843-851. 
\9 Bissler JJ, Siroky BJ, Yin H: Glomerulocystic kidney disease. Pediatr Nephrol 2010;25: 2049-2056; quiz 2056-2059.

10 Patel V, Li L, Cobo-Stark P, et al: Acute kidney injury and aberrant planar cell polarity induce cyst formation in mice lacking renal cilia. Hum Mol Genet 2008;17:1578-1590.

-11 de Chadarevian JP, Legido A, Miles DK, Katsetos CD: Epilepsy, atherosclerosis, myocardial infarction, and carbamazepine. J Child Neurol 2003;18:150-151.

12 Siroky B, Yin H, Bissler J: Insights into tuberous sclerosis complex renal disease. Pediatr Nephrol 2010, in press.

-13 O'Callaghan FJ, Harris T, Joinson C, et al: The relation of infantile spasms, tubers, and intelligence in tuberous sclerosis complex. Arch Dis Child 2004;89:530-533.

- 14 Ou YC, Wu HC, Yang CR, Chang CL, Hwang TI, Chang CH: Renal angiomyolipoma: experience of 23 patients. Chung Hua I Hsueh Tsa Chih (Taipei) 1991;48:217-223.

15 Bissler JJ, Racadio J, Donnelly LF, Johnson ND: Reduction of postembolization syndrome after ablation of renal angiomyolipoma. Am J Kidney Dis 2002;39:966-971.

16 Casper KA, Donnelly LF, Chen B, Bissler JJ: Tuberous sclerosis complex: renal imaging findings. Radiology 2002;225:451-456.

17 Adler J, Greweldinger J, Litzky G: 'Macro' aneurysm in renal angiomyolipoma: two cases, with therapeutic embolization in one patient. Urol Radiol 1984;6:201-203.

18 Kessler OJ, Gillon G, Neuman M, Engelstein $\mathrm{D}$, Winkler H, Baniel J: Management of renal angiomyolipoma: analysis of 15 cases. Eur Urol 1998;33:572-575.

19 Mouded IM, Tolia BM, Bernie JE, Newman HR: Symptomatic renal angiomyolipoma: report of 8 cases, 2 with spontaneous rup ture. J Urol 1978;119:684-688.

20 Pode D, Meretik S, Shapiro A, Caine M: Diagnosis and management of renal angiomyolipoma. Urology 1985;25:461-467.

21 Yamakado K, Tanaka N, Nakagawa T, Ko bayashi S, Yanagawa M, Takeda K: Renal angiomyolipoma: relationships between tumor size, aneurysm formation, and rupture. Radiology 2002;225:78-82.

22 Fox CH, Voleti V, Khan LS, Murray B, Vassalotti J: A quick guide to evidence-based chronic kidney disease care for the primary care physician. Postgrad Med 2008;120:E01E06.
23 Williams JM, Racadio JM, Johnson ND, Donnelly LF, Bissler JJ: Embolization of renal angiomyolipomata in patients with tuberous sclerosis complex. Am J Kidney Dis 2006;47:95-102.

24 Patel U, Simpson E, Kingswood JC, SaggarMalik AK: Tuberose sclerosis complex: analysis of growth rates aids differentiation of renal cell carcinoma from atypical or minimal-fat-containing angiomyolipoma. Clin Radiol 2005;60:665-673; discussion 3-4.

25 Eble JN, Amin MB, Young RH: Epithelioid angiomyolipoma of the kidney: a report of five cases with a prominent and diagnostically confusing epithelioid smooth muscle component. Am J Surg Pathol 1997;21:11231130.

26 Mai KT, Perkins DG, Collins JP: Epithelioid cell variant of renal angiomyolipoma. Histopathology 1996;28:277-280.

27 Karbowniczek M, Yu J, Henske EP: Renal angiomyolipomas from patients with sporadic lymphangiomyomatosis contain both neoplastic and non-neoplastic vascular structures. Am J Pathol 2003;162:491-500.

$\checkmark 28$ Lin CN, Chiang HS, Hsu SI, Huang AH, Chuang SS: Renal angiomyolipoma with a prominent angiomatous component and extramedullary hematopoiesis: a case report. Chung Hua I Hsueh Tsa Chih (Taipei) 1994; 53:185-187.

29 Tweeddale DN, Dawe CJ, McDonald JR: Angiolipoleiomyoma of the kidney: report of a case with observations on histogenesis. Cancer 1955;8:764-770.

30 Obuz F, Karabay N, Secil M, Igci E, Kovanlikaya A, Yorukoglu K: Various radiological appearances of angiomyolipomas in the same kidney. Eur Radiol 2000;10:897-899.

-31 Wong AL, McGeorge A, Clark AH: Renal angiomyolipoma: a review of the literature and a report of 4 cases. Br J Urol 1981;53:406-411.

32 Eble JN: Angiomyolipoma of kidney. Semin Diagn Pathol 1998;15:21-40.

33 Bjornsson J, Short MP, Kwiatkowski DJ, Henske EP: Tuberous sclerosis-associated renal cell carcinoma. Clinical, pathological, and genetic features. Am J Pathol 1996;149: 1201-1208.

34 Hardman JA, McNicholas TA, Kirkham N, Fletcher MS: Recurrent renal angiomyolipoma associated with renal carcinoma in a patient with tuberous sclerosis. Br J Urol 1993; 72:983-984.
35 Aydin H, Magi-Galluzzi C, Lane BR, et al: Renal angiomyolipoma: clinicopathologic study of 194 cases with emphasis on the epithelioid histology and tuberous sclerosis association. Am J Surg Pathol 2009;33:289297.

36 Saito K, Fujii Y, Kasahara I, Kobayashi N, Kasuga T, Kihara K: Malignant clear cell 'sugar' tumor of the kidney: clear cell variant of epithelioid angiomyolipoma. J Urol 2002;168: 2533-2534.

37 Pea M, Bonetti F, Martignoni G, et al: Apparent renal cell carcinomas in tuberous sclerosis are heterogeneous: the identification of malignant epithelioid angiomyolipoma. Am J Surg Pathol 1998;22:180-187.

38 Kim JK, Park SY, Shon JH, Cho KS: Angiomyolipoma with minimal fat: differentiation from renal cell carcinoma at biphasic helical CT. Radiology 2004;230:677-684.

39 Jinzaki M, Tanimoto A, Narimatsu Y, et al: Angiomyolipoma: imaging findings in lesions with minimal fat. Radiology 1997;205: 497-502.

-40 Hafron J, Fogarty JD, Hoenig DM, Li M, Berkenblit R, Ghavamian R: Imaging characteristics of minimal fat renal angiomyolipoma with histologic correlations. Urology 2005;66:1155-1159.

41 Mitchell DG, Crovello M, Matteucci T, Petersen RO, Miettinen MM: Benign adrenocortical masses: diagnosis with chemical shift MR imaging. Radiology 1992;185:345351.

-42 Israel GM, Hindman N, Hecht E, Krinsky G: The use of opposed-phase chemical shift MRI in the diagnosis of renal angiomyolipomas. AJR Am J Roentgenol 2005;184:18681872.

43 Young LR, Franz DN, Nagarkatte P, et al: Utility of [18F]2-fluoro-2-deoxyglucosePET in sporadic and tuberous sclerosis-associated lymphangioleiomyomatosis. Chest 2009;136:926-933.

44 Jiang X, Kenerson H, Aicher L, et al: The tuberous sclerosis complex regulates trafficking of glucose transporters and glucose uptake. Am J Pathol 2008;172:1748-1756.

-45 Bissler JJ, McCormack FX, Young LR, et al: Sirolimus for angiomyolipoma in tuberous sclerosis complex or lymphangioleiomyomatosis. N Engl J Med 2008;358:140-151. 